\title{
Fractional esterification rate of cholesterol in high-density lipoprotein associates with risk of coronary heart disease
}

Junmeng Liu', Ruiyue Yang ${ }^{2}$, Min Zhou' ${ }^{1}$, Wen Mao ${ }^{1}$, Hongxia Li ${ }^{2}$, Haijian Zhao ${ }^{3}$, Shu Wang ${ }^{2}$, Wenxiang Chen ${ }^{2,3}$, Jun Dong ${ }^{2^{*}}$ (D) and Qing He $\mathrm{1}^{1^{*}}$

\begin{abstract}
Background: Fractional esterification rate of cholesterol in high-density lipoprotein (FER $\mathrm{HDL}_{\mathrm{DL}}$ ) has been found to be closely correlated with atherosclerotic dyslipidemia, especially lipoprotein distributions, and is a potentially useful predictor for coronary heart disease (CHD). The associations of FER $\mathrm{HDL}_{\text {, }}$ measured by the simple and precise HPLC method, with angiographically defined CHD and its related risk factors in Chinese patients were evaluated.

Methods: Two hundred and fifty eight Chinese patients who had indications for angiography were enrolled in this study. Coronary angiograms were obtained by the standard techniques. FER $R_{H D L}$ was determined by the HPLC method. Cholesterol levels in serum HDL, LDL and subfractions were measured by ultracentrifugation/HPLC method. Associations between FER $\mathrm{HDL}_{\mathrm{L}}$ and $\mathrm{CHD}$ and $\mathrm{CHD}$ risk factors were analyzed.

Results: FER $_{H D L}$ was correlated with almost all the CHD risk factors. Compared with the non-CHD group, the CHD patients had higher values of FER $\mathrm{HDL}_{\mathrm{LL}}(20.9 \pm 7.9 \% / \mathrm{h}$ vs $17.7 \pm 7.1 \% / \mathrm{h}, p=0.001)$. FER $\mathrm{HDL}$ was found to be independently and positively correlated with $\log T G(\beta=0.386, P<0.001)$ and $\log (\operatorname{LDLb}-C)(\beta=0.165, P=0.020)$, respectively, and negatively correlated with $\log (\operatorname{HDL} 2-C)(\beta=-0.351, P<0.001)$. Logistic regression analysis demonstrated that age, diabetes mellitus, smoking and FER $\mathrm{HDL}_{\mathrm{L}}(\mathrm{OR}=1.056-1.080, p<0.05)$ were independent risk factors for CHD.

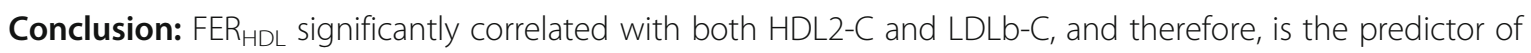
lipoprotein distributions. In addition, after correcting for the presence of classic risk factors, FER HDL was independently associated with the presence of angiographically defined CHD.
\end{abstract}

Keywords: Cholesterol, Esterification rate, High density lipoprotein, Lipoprotein subfractions, Coronary heart disease

\section{Background}

Epidemiologic and interventional studies have clearly established an inverse association between plasma levels of high-density lipoprotein (HDL) cholesterol (HDL-C) and incidence of coronary heart disease (CHD) $[1,2]$. However, contrary to what has been achieved in the field of low-density lipoprotein cholesterol (LDL-C) control through statin therapy, pharmacological modulation of HDL-C has not made a comparable success in the

\footnotetext{
*Correspondence: jun_dong@263.net; hegingli2001@yahoo.com

${ }^{2}$ The MOH Key Laboratory of Geriatrics, Beijing Hospital, National Center of Gerontology, Beijing 100730, People's Republic of China

${ }^{1}$ Cardiology Department, Beijing Hospital, Beijing 100730, People's Republic of China

Full list of author information is available at the end of the article
}

clinical arena. Several therapies, including nicotinic acid, fibric acid derivatives, and inhibitors of cholesteryl ester transfer protein (CETP), were not associated with CHD risk reduction despite significant increase in HDL-C levels [3]. In addition, there are several genetic syndromes of very low HDL-C that are not associated with an increased risk of premature CHD [4]. Therefore, the static measurement of HDL-C levels has inherent limitations as a metric of the functional effects of HDL and HDL associated CHD risks [5, 6]. The determination of HDL function, and not only HDL levels, may improve clinical CHD risk assessment, especially in lowrisk populations. 
Fractional esterification rate of HDL cholesterol $\left(\mathrm{FER}_{\mathrm{HDL}}\right.$ ) is defined as the percentage of HDL (after depleting the apoB-containing lipoproteins) free cholesterol (HDLFC) esterified during HDL incubation (ea. at $37{ }^{\circ} \mathrm{C}, 1 \mathrm{~h}$ ). In the last decades, researchers have found that FER $_{\mathrm{HDL}}$ is significantly correlated with atherosclerotic dyslipidemia and is a marker of lipoprotein particle sizes $[7,8]$. Subsequently, FER $_{\mathrm{HDL}}$ was shown to be a functional test of HDL quality and a strong predictor of positive findings on coronary angiography and therefore, is a potentially useful risk predictor for CHD [9-11].

FER $_{\text {HDL }}$ has been measured by an isotopic assay established by Dobiasova [12, 13]. Although this method is reliable, the time-consuming sample preparation and the requirement of radioactive isotope make it inconvenient for use in clinical laboratories. In our previous study, we established a simple, nonradioactive, and precise HPLC method for the measurement of FER $\mathrm{HDL}_{\text {[14]. With this }}$ method, we have documented significant associations between $F E R_{H D L}$ and lipid profiles, especially the negative correlation with large HDL and positive correlation with small-dense LDL concentrations in healthy volunteers. Therefore, the aims of the current study are to evaluate the associations of $\mathrm{FER}_{\mathrm{HDL}}$, measured by the HPLC method, with angiographically defined CHD and CHD severity, and the associations between FER $_{\mathrm{HDL}}$ and the major CHD risk factors in Chinese patients.

\section{Methods}

\section{Study subjects}

The study subjects included 258 patients hospitalized from 2008 to 2011 at Beijing Hospital, who had indications for angiography and had never received statin therapies in the preceding 6 months. Height, weight and blood pressure were measured. Baseline demographics and medical histories associated with $\mathrm{CHD}$, such as smoking, hypertension (HTN), and diabetes mellitus (DM) were recorded by selfreported questionnaire. The patients who were diagnosed with acute myocardial infarction, or receiving radiotherapy or chemotherapy were excluded from this study. Patients with systolic blood pressure (SBP) of $140 \mathrm{mmHg}$ or diastolic blood pressure (DBP) of $90 \mathrm{mmHg}$ or higher, or receiving antihypertensive therapy were considered to have HTN. Patients recorded for DM by self-report questionnaire or with a fasting blood glucose (FBG) concentration of $7.0 \mathrm{mmol} / \mathrm{L}$ were considered to have DM. Patients with a serum total cholesterol $(\mathrm{TC})>6.21 \mathrm{mmol} / \mathrm{L}$, LDL$\mathrm{C}>4.14 \mathrm{mmol} / \mathrm{L}$, triglycerides $(\mathrm{TG})>1.70 \mathrm{mmol} / \mathrm{L}$, or HDL-C $<1.04 \mathrm{mmol} / \mathrm{L}$, were considered to be dyslipidemia. Blood samples were collected after an overnight fast into common vacutainer tubes, serum was isolated within $2 \mathrm{~h}$, aliquoted and frozen at $-80^{\circ} \mathrm{C}$ until analyses.

\section{Ethics statement}

This study was reviewed and approved by the Beijing Hospital Ethics Committee. All studied individuals were informed in writing of the intended use of their samples and each provided written consent.

\section{Coronary angiography}

Coronary angiograms were obtained by the standard techniques with multiple views recorded. Coronary arteries were divided into 15 segments, according to the classification of the American Heart Association Grading Committee. Coronary artery segments were carefully selected by two cardiologists on the basis of smooth luminal borders and the absence of stenosis. The presence of stenosis was determined using a computer-assisted coronary angiography analysis system (Mipron 1; Kontron Co., Tokyo, Japan), and $\mathrm{CHD}$ was defined as the existence of any one of the coronary artery or major branch stenosis $\geq 50 \%$ in diameter. Individuals with $<50 \%$ stenosis were included into the non-CHD group. Individuals with negative findings in computed tomography of the coronary arteries or stress myocardial perfusion imaging were also included into the non-CHD group.

\section{Measurement of serum FER HDL $_{\text {by HPLC }}$}

Serum FER $_{\mathrm{HDL}}$ was measured using our previously reported HPLC method [13]. Briefly, serum samples were thawed at room temperature and $1 \mathrm{~mL}$ aliquots were transferred to another set of tubes. Subsequently, $0.1 \mathrm{~mL}$ of precipitation reagent $(10 \mathrm{~g} / \mathrm{L}$ solution of dextran sulfate and $0.5 \mathrm{~mol} / \mathrm{L} \mathrm{MgCl}_{2}$ ) was added, vortexed for $10 \mathrm{~s}$, incubated at room temperature for $15 \mathrm{~min}$, and then centrifuged at $1500 \mathrm{~g}$ at $4{ }^{\circ} \mathrm{C}$ for $30 \mathrm{~min}$. The HDL supernate was transferred and aliquoted into two tubes, with $0.4 \mathrm{~mL}$ per tube. One set of the tubes was placed in an ice water bath, while the other set was incubated at $37^{\circ} \mathrm{Cwater}$ bath for exactly $1 \mathrm{~h}$ and then put into ice water bath for HDLFC analyses. HDLFC was measured by our previously reported method $[14,15]$. Free cholesterol and internal standard (stigmasterol) were extracted with hexane, oxidized to cholest-4-en-3,6-diones with chromic acid, and analyzed by HPLC. FER $\mathrm{HDL}_{\text {w }}$ was presented by the percentage decrease of HDLFC mass during incubation at $37^{\circ} \mathrm{C}$ for $1 \mathrm{~h}$ and was calculated by the following equation:

$$
\operatorname{FER}_{\mathrm{HDL}}(\% / \mathrm{hr})=\left(\mathrm{HDLFC}_{0^{\circ} \mathrm{C}}-\mathrm{HDLFC}_{37^{\circ} \mathrm{C}}\right) / \mathrm{HDLFC}_{0^{\circ} \mathrm{C}} \times 100 \%
$$

\section{Other parameters and laboratory assays}

Cholesterol concentrations in serum HDL, LDL and subfractions (HDL-C, HDL2-C, HDL3-C, LDL-C, LDLa$\mathrm{C}$, and LDLb-C) as well as in lipoprotein (a) [Lp(a)-C] were measured by our previously established ultracentrifugation/HPLC methods $[16,17]$. Serum FBG, TC, TG, 
apolipoprotein (apo) CII, apoCIII, high-sensitive C reactive protein (hsCRP) and uric acid (URIC) were measured enzymatically using assay kits from Sekisui Medical Technologies (Osaka, Japan) on a Hitachi 7180 chemistry analyzer.

\section{Statistical analyses}

For continuous variables, normality was tested by Kolmogorow-Smirnov test. Variables with normal distributions were described as mean \pm standard deviation (SD). The means of variables were compared by T-test between two groups and by the one-way analysis of variance among multi-groups. The variables with skewed distribution were presented as median and interquartile ranges (25th to 75 th percentile). Categorical variables were presented as frequencies and percentages, and were analyzed by Chi-square test. Correlations between $\mathrm{FER}_{\mathrm{HDL}}$ and other parameters were analyzed by Spearman nonparametric test. Stepwise multiple linear regression analysis was used to test the independent relationships of $F E R_{H D L}$ with the measured variables. Meanwhile, collinearity testing was used to avoid including interdependent model variables. The significance levels for entering and removing an explanatory variable were set at 0.05 and 0.10 , respectively. The associations between measured variables and CHD were evaluated by multivariable logistic regression analysis. Odds ratios (ORs) for CHD versus non-CHD were estimated with the corresponding 95\% confidence intervals (CIs). The odds ratios were adjusted for Age, gender, body mass index (BMI), smoking status, and the presence or absence of DM, HTN, and dyslipidemia. All reported $P$ values were two-tailed, with a $P$ value of 0.05 indicating statistical significance. Statistical analyses were performed with the use of SPSS software, version 17.0 (SPSS Inc.).

\section{Results \\ Demographic and clinical characteristics of the study population}

The study population consisted of 258 hospitalized patients, 170 men and 88 women, aged $30 \sim 84$ years old. The Demographic and clinical characteristics were shown in Table 1. These subjects were divided into CHD and non-CHD groups according to coronary angiography results. The mean age was $64.2 \mathrm{y}$ in the $\mathrm{CHD}$ group and $61.9 \mathrm{y}$ in the non-CHD group $(P=0.09)$. There were significantly more males, higher prevalence of DM, and higher percentage of smoking in CHD cases than in the controls. Age, BMI, blood pressure, and percentage of HTN and dyslipidemia were not significantly different between the two groups.
Table 1 Demographic and clinical characteristics of study population

\begin{tabular}{llll}
\hline & $\begin{array}{l}\text { Non CHD group } \\
(n=100)\end{array}$ & $\begin{array}{l}\text { CHD group } \\
(n=158)\end{array}$ & $P$ \\
\hline Age, years & $61.9 \pm 10.6$ & $64.2 \pm 10.8$ & 0.091 \\
BMI, kg/m² & $25.6 \pm 3.6$ & $25.7 \pm 3.1$ & 0.655 \\
SBP, mmHg & $128.0 \pm 16.4$ & $130.8 \pm 17.3$ & 0.191 \\
DBP, mmHg & $76.4 \pm 10.9$ & $74.8 \pm 9.9$ & 0.248 \\
Men, \% & $58(58.0)$ & $112(70.9)$ & 0.033 \\
Smoker, \% & $36(36.0)$ & $77(48.7)$ & 0.045 \\
Hypertension, \% & $62(62.0)$ & $110(69.6)$ & 0.206 \\
Diabetes, \% & $17(17.0)$ & $59(37.3)$ & $<0.001$ \\
Dyslipidemia, \% & $72(72.0)$ & $129(81.6)$ & 0.069 \\
\hline
\end{tabular}

\section{Univariate analyses of laboratory indexes between CHD} and non-CHD groups

Results of univariate analyses of laboratory parameters between $\mathrm{CHD}$ and non-CHD groups were shown in Table 2. There were no significant differences for serum TC, LDL-C, LDLa-C, LDLb-C, HDL3-C, and Lp(a)-C concentrations between the two groups. Compared to the non-CHD group, the CHD group had significantly higher levels of TG and lower levels of HDL-C and HDL2-C. Serum FBG, apoCII, apoCIII, URIC and hsCRP levels were significantly higher in CHD group than in the non CHD group. FER $\mathrm{HDL}_{\text {L }}$ was found to be significantly higher in CHD group than in the controls $(20.8 \pm 7.9$ vs $17.7 \pm 7.1 \% / \mathrm{h}, P=0.001)$. In addition, the

Table 2 Univariate analyses of laboratory indexes between two groups

\begin{tabular}{|c|c|c|c|}
\hline & $\begin{array}{l}\text { Non CHD group } \\
(n=100)\end{array}$ & $\begin{array}{l}\text { CHD group } \\
(n=158)\end{array}$ & $P$ \\
\hline$\overline{\mathrm{TC}, \mathrm{mmol} / \mathrm{L}}$ & $4.55 \pm 0.87$ & $4.60 \pm 0.98$ & 0.662 \\
\hline $\mathrm{TG}^{\mathrm{a}}, \mathrm{mmol} / \mathrm{L}$ & $1.30(1.00 \sim 1.62)$ & $1.60(1.20 \sim 2.20)$ & $<0.001$ \\
\hline LDL-C ${ }^{a}, \mathrm{mmol} / \mathrm{L}$ & $2.45(2.12 \sim 2.96)$ & $2.33(1.94 \sim 2.95)$ & 0.483 \\
\hline LDLa-C, mmol/L & $2.17 \pm 0.65$ & $2.11 \pm 0.73$ & 0.285 \\
\hline LDLb-C ${ }^{\mathrm{a}}, \mathrm{mmol} / \mathrm{L}$ & $0.26(0.19 \sim 0.38)$ & $0.28(0.19 \sim 0.46)$ & 0.350 \\
\hline $\mathrm{HDL}-\mathrm{C}^{\mathrm{a}}, \mathrm{mmol} / \mathrm{L}$ & $1.03(0.88 \sim 1.25)$ & $0.96(0.82 \sim 1.18)$ & 0.017 \\
\hline $\mathrm{HDL} 2-\mathrm{C}^{\mathrm{a}}, \mathrm{mmol} / \mathrm{L}$ & $0.55(0.43 \sim 0.72)$ & $0.50(0.40 \sim 0.65)$ & 0.022 \\
\hline $\mathrm{HDL} 3-\mathrm{C}^{\mathrm{a}}, \mathrm{mmol} / \mathrm{L}$ & $0.48(0.43 \sim 0.53)$ & $0.46(0.40 \sim 0.52)$ & 0.063 \\
\hline $\mathrm{Lp}(\mathrm{a})-\mathrm{C}^{\mathrm{a}}, \mathrm{mmol} / \mathrm{L}$ & $0.06(0.03 \sim 0.11)$ & $0.06(0.04 \sim 0.11)$ & 0.559 \\
\hline Apo $C \|^{a}, \mathrm{mg} / \mathrm{dL}$ & $3.80(2.40 \sim 4.80)$ & $4.10(2.80 \sim 5.45)$ & 0.046 \\
\hline Apo CIII ${ }^{a}, \mathrm{mg} / \mathrm{dL}$ & $7.50(6.20 \sim 9.10)$ & $8.50(6.60 \sim 10.50)$ & 0.033 \\
\hline hsCRP ${ }^{a}, \mathrm{mg} / \mathrm{dL}$ & $0.12(0.05 \sim 0.24)$ & $0.18(0.08 \sim 0.28)$ & 0.033 \\
\hline $\mathrm{FBG}{ }^{\mathrm{a}}, \mathrm{mmol} / \mathrm{L}$ & $5.00(4.70 \sim 5.60)$ & $5.30(4.88 \sim 6.10)$ & 0.028 \\
\hline URIC ${ }^{a}$, umol/L & $329.0(260.0 \sim 378.0)$ & $355.0(306.5 \sim 412.5)$ & 0.002 \\
\hline $\mathrm{FER}_{\mathrm{HDL}} \%$ \%/h & $17.7 \pm 7.1$ & $20.8 \pm 7.9$ & 0.001 \\
\hline
\end{tabular}

For apoCII and apoCIII, 125 cases and 75 controls were analyzed ${ }^{\text {a }}$ Median (Q1 Q3) 
158 CHD patients were further divided into 1-vessle $(n=51)$, 2-vessel $(n=44)$ and 3-vessel $(n=63)$ stenosed subgroups and the above parameters were compared among groups. As shown in Fig. 1, with the increase of the number of stenosed vessels, $\mathrm{FER}_{\mathrm{HDL}}$ was significantly increased $(P=0.004)$.

\section{Correlations between $F E R_{\mathrm{HDL}}$ and other parameters}

Univariate correlations between $\mathrm{FER}_{\mathrm{HDL}}$ and clinical and laboratory parameters were analyzed and the results were shown in Table 3 . FER $\mathrm{HDL}_{\mathrm{H}}$ values were significantly and positively correlated with BMI $(P<0.001)$, serum FBG $(P<0.001)$, TG $(P<0.001)$, LDLb-C $(P<0.001)$, apoCII $(P<0.001)$, apoCIII $(P<0.001)$, Lp(a) $(P=0.005)$, hsCRP $(P=0.001)$, and URIC $(P<0.001)$, respectively. On the other hand, significantly negative correlations between FER $\mathrm{HDL}_{\mathrm{HL}}$ and age $(P<0.001)$, HDLC $(P<0.001)$, HDL2-C $(P<0.001)$ HDL3-C $(P<0.001)$ and LDLa-C $(P=0.005)$ were observed respectively.

\section{Multiple linear regression model}

For multivariate reevaluation of the univariate correlations, all variables given in Table 3 were entered into a stepwise multiple linear regression analysis as independent variables to identify significant contributors to the distribution of $\mathrm{FER}_{\mathrm{HDL}}$. The relationships between FER $_{\text {HDL }}$ and other CHD risk factors were shown in Table 4. FER $\mathrm{HDL}_{\mathrm{L}}$ was found to be independently and positively correlated with $\log$ TG $(\beta=0.386, P<0.001)$, and $\log ($ LDLb-C $)(\beta=0.165, P=0.020)$, respectively, and negatively correlated with age $(\beta=-0.152$, $P=0.017)$ and $\log ($ HDL2-C $)(\beta=-0.351, P<0.001)$.

\section{Logistic regression model}

Logistic regression analysis was carried out to further quantify the association between $\mathrm{FER}_{\mathrm{HDL}}$ and $\mathrm{CHD}$, and the results were shown in Table 5. Multivariate

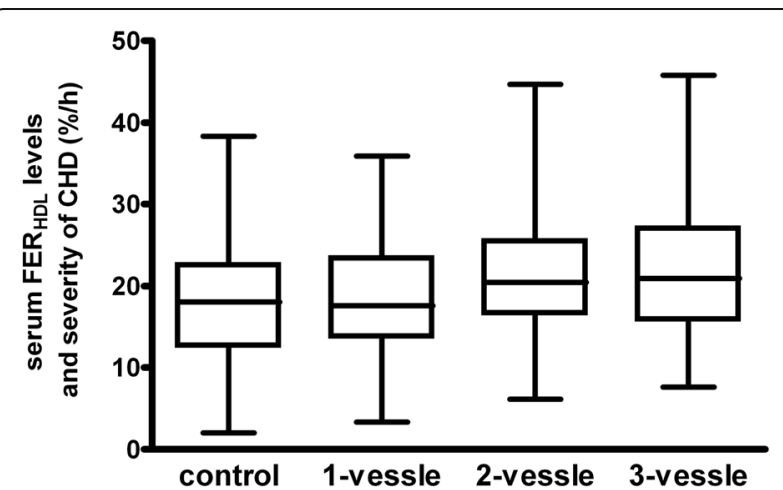

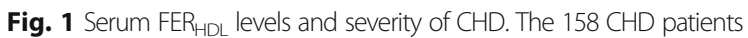
were divided into 1-vessle $(n=51), 2$-vessel $(n=44)$ and 3-vessel $(n=63)$ stenosed subgroups. The box plots show the median and 25th and 75th percentiles. Whiskers in the plots represent the highest and lowest values
Table 3 Univariate correlations of FER $\mathrm{HDL}_{\mathrm{L}}$ with clinical and laboratory parameters

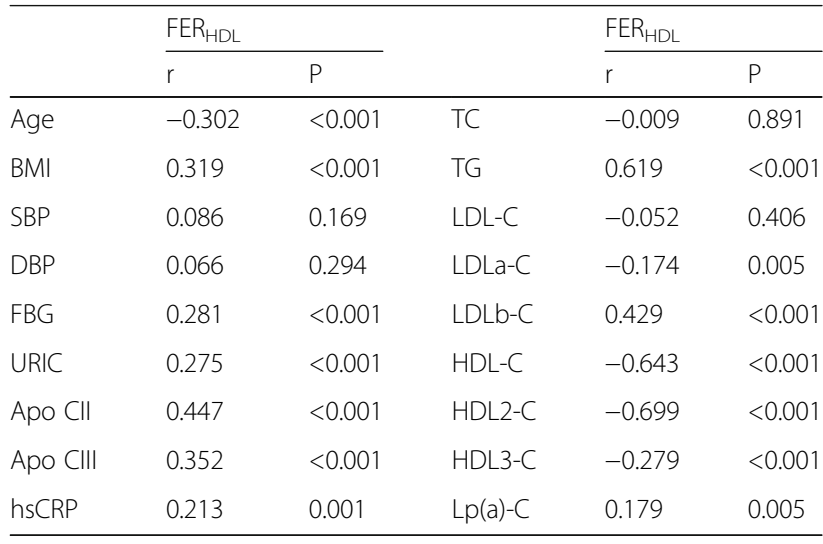

adjustment was used to control for traditional CHD risk factors, including age, gender, BMI, smoking, DM, hypertension, and dyslipidemia. After this multivariate adjustment, each $1 \mathrm{SD}$ increase in $\mathrm{FER}_{\mathrm{HDL}}$ value was associated with an approximately two-fold increase in the risk of $\mathrm{CHD}(\mathrm{OR}=1.716,95 \% \mathrm{CI}: 1.254-2.348$, $P<0.001)$. As compared with subjects in the lowest quartile of $\mathrm{FER}_{\mathrm{HDL}}$ levels, the OR (95\% CIs) for CHD risk in subjects belonging to quartiles 2,3 , and 4 were 2.447 (1.150-5.204), 1.553 (0.734-3.282), and 3.174 $(1.408-7.155)$, respectively $(P$ trend $=0.022)$. Age, smoking and DM were also independently correlated with the risk of $\mathrm{CHD}$, and $\mathrm{FER}_{\mathrm{HDL}}$ was the only lipid related risk factor for CHD.

\section{Discussion}

FER $_{\mathrm{HDL}}$ measures the rate of HDL cholesterol esterification which is catalyzed by the Lecithin:cholesterolacyl transferase (LCAT) in vitro. LCAT is a plasma enzyme that esterifies free cholesterol, primarily at the surface of the HDL particle, after which the cholesteryl ester molecules migrate into the inner core of the lipoprotein for metabolism [18]. As an important enzyme involved in the reverse cholesterol transport (RCT), LCAT plays a key role in the maturation of HDL particles and the

Table 4 Multiple linear regression analysis with FER $\mathrm{HDL}_{\mathrm{H}}$ as the dependent variable

\begin{tabular}{lllll}
\hline Variables & Regression coefficient & SE & $\beta$ & $P$ \\
\hline Age & -0.093 & 0.038 & -0.152 & 0.017 \\
Log (HDL2-C) & -19.259 & 3.653 & -0.351 & $<0.001$ \\
Log (LDLb-C) & 4.326 & 1.832 & 0.165 & 0.020 \\
Log TG & 16.037 & 2.746 & 0.386 & $<0.001$
\end{tabular}

$\beta$ indicates the standardized partial regression coefficient. Adjusted $\mathrm{R}^{2}=0.453, P=0.017$

All variables given in Table 3 were entered into a stepwise multiple linear regression analysis as independent variables. Collinearity testing was used to avoid including interdependent model variables. $P$ values for entry and removal, 0.05 and 0.10 , respectively 
Table $\mathbf{5}$ Logistic regression model for the case-control study

\begin{tabular}{|c|c|c|c|c|}
\hline & \multicolumn{2}{|l|}{ Univariable } & \multicolumn{2}{|l|}{ Multivariable $^{a}$} \\
\hline & OR $(95 \% \mathrm{Cl})$ & $P$ & OR $(95 \% \mathrm{Cl})$ & $P$ \\
\hline Age & $1.242(0.965 \sim 1.599)$ & 0.092 & $1.646(1.203 \sim 2.252)$ & 0.002 \\
\hline Smoke & $1.298(1.005 \sim 1.676)$ & 0.045 & $1.451(1.083 \sim 1.943)$ & 0.013 \\
\hline $\mathrm{DM}$ & $1.638(1.232 \sim 2.177)$ & 0.001 & $1.576(1.177 \sim 2.110)$ & 0.002 \\
\hline \multicolumn{5}{|c|}{$\mathrm{FER}_{\mathrm{HDL}}$ as a continuous variable } \\
\hline Per SD increment & $1.556(1.181 \sim 2.050)$ & 0.002 & $1.716(1.254 \sim 2.348)$ & 0.001 \\
\hline \multicolumn{5}{|c|}{$\mathrm{FER}_{\text {HDL }}$ as a categorical variable } \\
\hline First quartile & 1.0 (referent) & - & 1.0 (referent) & - \\
\hline Second quartile & $2.230(1.092 \sim 4.557)$ & 0.028 & $2.447(1.150 \sim 5.204)$ & 0.020 \\
\hline Third quartile & $1.285(0.641 \sim 2.573)$ & 0.480 & $1.553(0.734 \sim 3.282)$ & 0.249 \\
\hline Fourth quartile & $2.943(1.404 \sim 6.170)$ & 0.004 & $3.174(1.408 \sim 7.155)$ & 0.005 \\
\hline$P$-value for trend & 0.015 & & 0.022 & \\
\hline
\end{tabular}

a Set of independent variables: Age, gender, BMI, smoking, diabetes mellitus, hypertension, dyslipidemia and FER $\mathrm{HDL}_{\text {levels }}$

RCT process [19]. Studies have suggested that the preferred substrates for LCAT are Pre $\beta$ - and small sizedHDL, and the smaller the HDL particles, the faster their surface free cholesterol esterified, and FER $_{\mathrm{HDL}}$ reflects HDL particle distributions $[10,11]$. The relationship between $\mathrm{FER}_{\mathrm{HDL}}$ and LDL particle size is currently unknown. However, the metabolism of HDL and LDL are closely connected, and TG might be an important mediator between $\mathrm{HDL}$ and LDL. Therefore, FER $\mathrm{HDL}_{\mathrm{H}}$ not only reflects LCAT activity in specific conditions, but also reflects the maturation of HDL, the balance between uptake of cholesterol and transport of cholesterol esters, as well as the efficiency of RCT. Unlike the static HDL-C levels, FER $_{\text {HDL }}$ might serve as a functional test of lipoprotein quality and HDL metabolism. Although the associations of $\mathrm{FER}_{\mathrm{HDL}}$ with lipoprotein distribution and CHD risks have been quite consistent in previous studies, clinical application of $\mathrm{FER}_{\mathrm{HDL}}$ was hindered by the tedious and radioactive assay method. In our previous study, we reported a simple, precise, and nonradioactive method for the measurement of $\mathrm{FER}_{\mathrm{HDL}}$, and confirmed the relationship between FER $_{\text {HDL }}$ and lipid profiles in apparently healthy subjects [14]. In the present study, we investigated the associations of serum FER $_{\text {HDL }}$ with angiographically defined CHD, CHD severity and CHD risk factors in Chinese patients. As statin therapy significantly affects lipid profiles, the use of statins was strictly excluded in our study.

We first analyzed the correlations of FER $\mathrm{HDL}_{\text {with }}$ classic CHD risk factors and several other variables, and found that $\mathrm{FER}_{\mathrm{HDL}}$ was positively correlated with male gender, smoking, BMI, serum FBG, TG, and negatively correlated with HDL-C, which was in accordance with previous findings $[13,14]$. HDL and LDL are highly heterogeneous, and the concept that certain subfractions may be better predictors of $\mathrm{CHD}$ risk is of great concern. Many population studies have suggested that large HDL2 may be more cardioprotective than smaller sized HDL3 [20, 21]. However, there are also inconsistencies, with reports suggesting that HDL2 and HDL3 are equally cardioprotective [22]. In our study, FER HDL $_{\text {L }}$ showed significantly negative correlation with both HDL2-C and HDL3-C, in which correlation with HDL2$\mathrm{C}$ remained significant in multiple linear regression models. Prospective studies have reported the small LDL phenotype to be an important predictor for subsequent cardiovascular diseases [23, 24]. In accordance with previous findings, a significantly positive correlation between FER $_{\text {HDL }}$ and LDLb-C, but not LDLa-C, was observed. These results confirmed that $\mathrm{FER}_{\mathrm{HDL}}$ is a reflection of lipoprotein subfraction distributions. Furthermore, FER $_{\mathrm{HDL}}$ was also positively associated with BMI, FBG, Uric acid, apoCII, apoCII, and hsCRP. These results suggested that $\mathrm{FER}_{\mathrm{HDL}}$ is associated with almost all known CHD risk factors, and is a potentially valuable risk factor for predicting atherosclerosis and CHD.

In contrary with some of the previous studies, negative correlation between serum FER $_{\mathrm{HDL}}$ and age, as well as TG and age $(r=-0.157, p<0.05)$, was observed. In this study, $90 \%$ of the subjects were above $50 \mathrm{y}$ and there were more males than females. Our previous investigation showed that TG levels in Chinese males, but not in females, decreased after age of 50y [25], which might be associated with decreased $\mathrm{FER}_{\mathrm{HDL}}$ values in males. The negative correlation found between $\mathrm{FER}_{\mathrm{HDL}}$ and age may have resulted, by some extent, from the averagely old age and gender differences. Significantly positive correlation between $\mathrm{FER}_{\mathrm{HDL}}$ and age was observed in our previous study with healthy volunteers from age 19-72 [14].

We have shown that individuals with positive angiographic findings tended to be older, smoking, with higher prevalence of $\mathrm{DM}$ and higher $\mathrm{FER}_{\mathrm{HDL}}$ levels. In 
this study, logistic regression analysis showed that $\mathrm{FER}_{\mathrm{HDL}}$, age, DM and smoking were independent risk factors for angiographically proven $\mathrm{CHD}$, and $\mathrm{FER}_{\mathrm{HDL}}$ was the only lipid related risk factor for CHD. These findings were consistent with those found by previous reports although our study sample is apparently different in ethnicity, BMI, and probably other culture-related aspects, such as lifestyle and diet. In addition, we also found that $\mathrm{FER}_{\mathrm{HDL}}$ was associated with the severity of CHD.

The potential mechanisms involved in the relationship of $\mathrm{FER}_{\mathrm{HDL}}$ and lipid profiles are not clear. If high FER $_{\text {HDL }}$ does indeed reflect a high endogenous esterification rate in the HDL fraction, this finding disagrees with the generally accepted opinion that LCAT activity is beneficial to the process of RCT. Another possible explanation is that high $\mathrm{FER}_{\mathrm{HDL}}$ may reflect low endogenous LCAT activity. The potential mechanism is that, due to some adverse effect (e.g. lipoproteins), endogenous LCAT activity is low, and as a result, small HDL particles accumulate. When leaving these unfavorable factors (in vitro HDL fraction), LCAT activity increases due to sufficient amount of small HDL particles. This hypothesis requires further investigation.

The following limitations of our study should be considered. First, the control subjects were defined as patients with $<50 \%$ narrowing of their coronary arteries. These control subjects generally had higher CHD risks and were not truly healthy individuals, which may have underestimated the association between FER $_{\mathrm{HDL}}$ and CHD and limited the power of this study. Second, although our results remained consistent after multiple adjustments, we cannot exclude the possibility of residual confounding because some information was not recorded, including family history of $\mathrm{CHD}$, lifestyle, stress, and other possible risk factors for CHD. Third, since this study was a case-control design, the findings need to be confirmed in future prospective studies.

\section{Conclusions}

In conclusion, by using a simple and nonradioactive HPLC method, we demonstrated that FER $\mathrm{HDL}_{\text {signifi- }}$ cantly correlated with both HDL2-C and LDLb-C, and therefore, is a predictor of lipoprotein distributions. After correcting for the presence of classic risk factors and several other variables, FER $_{\mathrm{HDL}}$ was found to be independently associated with the presence of angiographically proven $\mathrm{CHD}$.

\footnotetext{
Abbreviations

CHD: Coronary heart disease; DM: Diabetes mellitus; FBG: Fasting blood glucose; $F E R_{H D L}$ : Fractional esterification rate of cholesterol in high density lipoprotein; HDL2: High-density lipoprotein of d 1.063-1.125 kg/L; HDL3: High-density lipoprotein of d 1.125-1.210 kg/L; HDL-C: HDLcholesterol; HDLFC: HDL free cholesterol; HTN: Hypertension; LDLa: Largebuoyant LDL; LDLb: Small-dense LDL; LDL-C: LDL-cholesterol; URIC: Uric acid
}

\section{Acknowledgements}

This study was supported by research grants from National Natural Science Foundation of China $(30872413,81472035)$.

\section{Funding}

This study was supported by research grants from National Natural Science Foundation of China $(30872413,81472035)$.

\section{Availability of data and materials}

The datasets used and/or analysed during the current study are available from the corresponding author on reasonable request.

\section{Authors' contributions}

Conceived and designed the experiments: JL WC JD QH. Performed the experiments: JL RY MZ WM HL HZ SW JD. Analyzed the data: JL RY JD QH. Wrote the paper: JL RY JD QH. Reviewed manuscript and made suggestions: JL RY WC JD QH. All authors read and approved the final manuscript.

\section{Authors' information}

Not applicable.

\section{Ethics approval and consent to participate}

This study was reviewed and approved by the Beijing Hospital Ethics Committee. All studied individuals were informed in writing of the intended use of their samples and each provided written consent.

\section{Consent for publication}

Not applicable.

\section{Competing interests}

No authors declared any potential conflicts of interest. The funding organizations played no role in the design of the study, review and interpretation of data, or preparation or approval of the manuscript.

\section{Publisher's Note}

Springer Nature remains neutral with regard to jurisdictional claims in published maps and institutional affiliations.

\section{Author details}

${ }^{1}$ Cardiology Department, Beijing Hospital, Beijing 100730, People's Republic of China. ${ }^{2}$ The MOH Key Laboratory of Geriatrics, Beijing Hospital, National Center of Gerontology, Beijing 100730, People's Republic of China. ${ }^{3}$ Beijing Hospital and National Center for Clinical Laboratories, Beijing 100730, People's Republic of China.

Received: 30 May 2017 Accepted: 2 August 2017

Published online: 24 August 2017

\section{References}

1. Castelli WP, Garrison RJ, Wilson PW, Abbott RD, Kalousdian S, Kannel WB. Incidence of coronary heart disease and lipoprotein cholesterol levels. The Framingham Study. JAMA. 1986:256:2835-8.

2. Nicholls SJ, Tuzcu EM, Sipahi I, Grasso AW, Schoenhagen P, Hu T, Wolski K, Crowe T, Desai MY, Hazen SL, Kapadia SR, Nissen SE. Statins, high-density lipoprotein cholesterol, and regression of coronary atherosclerosis. JAMA. 2007;297:499-508

3. Briel M, Ferreira-Gonzalez I, You JJ, Karanicolas PJ, AkI EA, Wu P, Blechacz B, Bassler D, Wei X, Sharman A, Whitt I, Alves da Silva S, Khalid Z, Nordmann AJ, Zhou Q, Walter SD, Vale N, Bhatnagar N, O'Regan C, Mills EJ, Bucher HC, Montori VM, Guyatt GH. Association between change in high density lipoprotein cholesterol and cardiovascular disease morbidity and mortality: systematic review and meta-regression analysis. BMJ. 2009;338:b92.

4. Kunnen S, Van Eck M. Lecithin:cholesterol acyltransferase: old friend or foe in atherosclerosis? J Lipid Res. 2012;53:1783-99.

5. Karathanasis SK, Freeman LA, Gordon SM, Remaley AT. The changing face of HDL and the best way to measure it. Clin Chem. 2017:63:196-210.

6. Fisher EA, Feig JE, Hewing B, Hazen SL, Smith JD. High-density lipoprotein function, dysfunction, and reverse cholesterol transport. Arterioscler Thromb Vasc Biol. 2012;32:2813-20.

7. Ohta T, Saku K, Takata K, Nagata N, Maung KK, Matsuda I. Fractional esterification rate of cholesterol in high density lipoprotein $(\mathrm{HDL})$ can 
predict the particle size of low density lipoprotein and HDL in patients with coronary heart disease. Atherosclerosis. 1997;135:205-12.

8. Dobiásová M, Urbanová Z, Samánek M. Relations between particle size of HDL and LDL lipoproteins and cholesterol esterification rate. Physiol Res. 2005:54:159-65.

9. Frohlich J, Dobiásová M. Fractional esterification rate of cholesterol and ratio of triglycerides to HDL-cholesterol are powerful predictors of positive findings on coronary angiography. Clin Chem. 2003;49:1873-80.

10. Dobiásová M, Frohlich J, Sedová M, Cheung MC, Brown BG. Cholesterol esterification and atherogenic index of plasma correlate with lipoprotein size and findings on coronary angiography. J Lipid Res. 2011;52:566-71.

11. Dobiášová M. Atherogenic impact of lecithin-cholesterol acyltransferase and its relation to cholesterol esterification rate in $\mathrm{HDL}(\mathrm{FER}(\mathrm{HDL}))$ and AIP [log(TG/HDL-C)] biomarkers: the butterfly effect? Physiol Res. 2017:4(66):193-203.

12. Dobiásová M, Frohlich J. Measurement of fractional esterification rate of cholesterol in plasma depleted of apoprotein B containing lipoprotein: methods and normal values. Physiol Res. 1996;45:65-73.

13. Dobiásová M, Adler L, Ohta T, Frohlich J. Effect of labeling of plasma lipoproteins with $\left[{ }^{3} \mathrm{H}\right]$ cholesterol on values of esterification rate of cholesterol in apolipoprotein B depleted plasma. J Lipid Res. 2000:41:1356-7.

14. Dong J, Yu S, Yang R, Li H, Guo H, Zhao H, Wang S, Chen W. A simple and precise method for direct measurement of fractional esterification rate of high density lipoprotein cholesterol by high performance liquid chromatography. Clin Chem Lab Med. 2014;52:557-64.

15. Dong J, Chen W, Wang S, Zhang J, Li H, Guo H, Man Y, Chen B. Jones oxidation and high performance liquid chromatographic analysis of cholesterol in biological samples. J Chromatogr B. 2007;15:239-46.

16. Dong J, Guo H, Yang R, Li H, Wang S, Zhang J, Chen W. Serum LDL- and HDL-cholesterol determined by ultracentrifugation and HPLC. J Lipid Res. 2011;52:383-8.

17. Dong J, Guo H, Yang R, Li H, Wang S, Zhang J, Zhou W, Chen W. A novel and precise method for simultaneous measurement of serum HDL and LDL subfractions and lipoprotein (a) cholesterol by ultracentrifugation and highperformance liquid chromatography. Clin Chim Acta. 2012;413:1071-6.

18. Calabresi L, Franceschini G. Lecithin:cholesterol acyltransferase, high-density lipoproteins, and atheroprotection in humans. Trends Cardiovasc Med. 2010; 20:50-3.

19. Ossoli A, Simonelli S, Vitali C, Franceschini G, Calabresi L. Role of LCAT in atherosclerosis. J Atheroscler Thromb. 2016;23:119-27.

20. Dobiasova M, Stribrna J, Pritchard PH, Frohlich JJ. Cholesterol esterification rate in plasma depleted of very low and low density lipoproteins is controlled by the proportion of HDL2 and HDL3 subclasses: study in hypertensive and normal middle-aged and septuagenarian men. J Lipid Res. 1992:33:1411-8

21. Morgan J, Carey C, Lincoff A, Capuzzi D. High-density lipoprotein subfractions and risk of coronary artery disease. Curr Atheroscler Rep. 2004; 6:359-65.

22. Williams PT, Feldman DE. Prospective study of coronary heart disease vs. HDL2, HDL3, and other lipoproteins in Gofman's Livermore cohort. Atherosclerosis. 2011;214:196-202.

23. Koba S, Yokota Y, Hirano T, Ito Y, Ban Y, Tsunoda F, Sato T, Shoji M, Suzuki H, Geshi E, Kobayashi Y, Katagiri T. Small LDL-cholesterol is superior to LDLcholesterol for determining severe coronary atherosclerosis. J Atheroscler Thromb. 2008;15:250-60.

24. Diffenderfer MR, Schaefer EJ. The composition and metabolism of large and small LDL. Curr Opin Lipidol. 2014;25:221-6.

25. Li JZ, Wang S, Dong J, Zhu LH, Li YL, Gao DL, Tian YP, Dong L, Yang L, Li SK. Present status of serum lipid levels in Beijing professional populations and its trend of changes over 15 years-a collaborative study of seven research and clinical laboratories in Beijing. Clin Chim Acta. 2005;352:199-207.

\section{Submit your next manuscript to BioMed Central and we will help you at every step:}

- We accept pre-submission inquiries

- Our selector tool helps you to find the most relevant journal

- We provide round the clock customer support

- Convenient online submission

- Thorough peer review

- Inclusion in PubMed and all major indexing services

- Maximum visibility for your research

Submit your manuscript at www.biomedcentral.com/submit
Biomed Central 